\title{
Aquired Aorto-Atrial Fistula from Intravenous Drug Use with Underlying Congenital Heart Disease
}

\author{
Mohamed Tashani, MD', Laura Given, MD $^{2}$, \\ Emhemmid Karem, $\mathbf{M D}^{3}$, and Mahmoud Abualayem, MD $^{3}$
}

Author affiliations are listed at the end of this

The development of an aorta-atrial fistula secondary to mechanical aortic valve infective endocarditis is a rare, serious complication. The fistula is an aberrant intra-cardiac shunt that occurs between the aorta and either the left or right atrium. An aorta-atrial fistula can be congenital or acquired. In the case of infective endocarditis, the infection's expansion beyond the valvular structure may result in an aorto-cavitary fistula (ACF or AAF) with an estimated incidence of $1-2 \% .1$ No clinical trials have been conducted for the best approach of management for this condition. Therefore, treatment strategies are applied on a case-by-case basis by expert opinion. Patients develop symptoms of heart failure secondary to AAF complications. The underlying cause of AAF needs to be identified with the use of imaging studies to determine the approach of optimal treatment.2 We are reporting a case of a 32-year-old male with a history of repaired congenital heart disease, who developed mechanical aortic valve infective endocarditis from intravenous drug use with subsequent development of an aorta-atrial fistula.

article.

Correspondence to: Mohamed Tashani, MD Saint Mary's Medical Center mohamed.tashani@st-marys.org

\section{KEYWORDS}

AAF, Infective Endocarditis, Prosthetic Valve, Congenital Heart Disease

A 32-year-old male with a past medical history of congenital aortic stenosis presented to the hospital with two weeks of fever. At the age of 8 , he underwent open aortic valvotomy with resection of a subaortic ring and myomectomy. Three years prior to the current presentation, he was diagnosed with severe aortic insufficiency and underwent re-entry median sternotomy, resection of the subaortic ring, left ventricular septal myotomy-myomectomy, reconstruction of left ventricular outflow tract with On-X 23 $\mathrm{mm}$ Dacron valve conduit, re-implantation of left and right coronary ostia, replacement of the ascending aorta, and tricuspid valve repair using a $32 \mathrm{~mm}$ triad annuloplasty ring procedure. Gross description in the operative report disclosed findings of a bicuspid structural valve. On the pathology report, fibromyxoid degenerative changes of the valvular leaflets were found.

At presentation to the outside facility, he had complaints of bilateral lower extremity weakness, vision changes, fever, chills, myalgias, and malaise for two weeks. Per patient report, he had used suboxone intravenously. The TEE showed an abscess with complete destruction of the left ventricular outflow track with a shunt of the LVOT to the left atrium, destruction of the aortic root, and a large anterior leaflet aortic valve vegetation. The $C T$ head revealed bilateral occipital lobe hemorrhages and a large $3.7 \mathrm{~cm}$ left occipital lobe hematoma. There was mass-effect and cerebral edema, but no midline shift. Therefore, he was diagnosed with an ischemic stroke due to septic emboli with hemorrhagic conversion. His INR was supratherapeutic at 5.9, which was reversed after receiving KCENTRA and Vitamin K. He was seen by neurosurgery who recommended no surgical intervention and to hold anticoagulation. CT chest reported evidence of systemic embolization. Vancomycin was started with rifampin for synergy. The patient was transferred from the outside hospital to a higher level of care for evaluation by cardiothoracic surgery after being diagnosed with complicated MRSA mechanical 


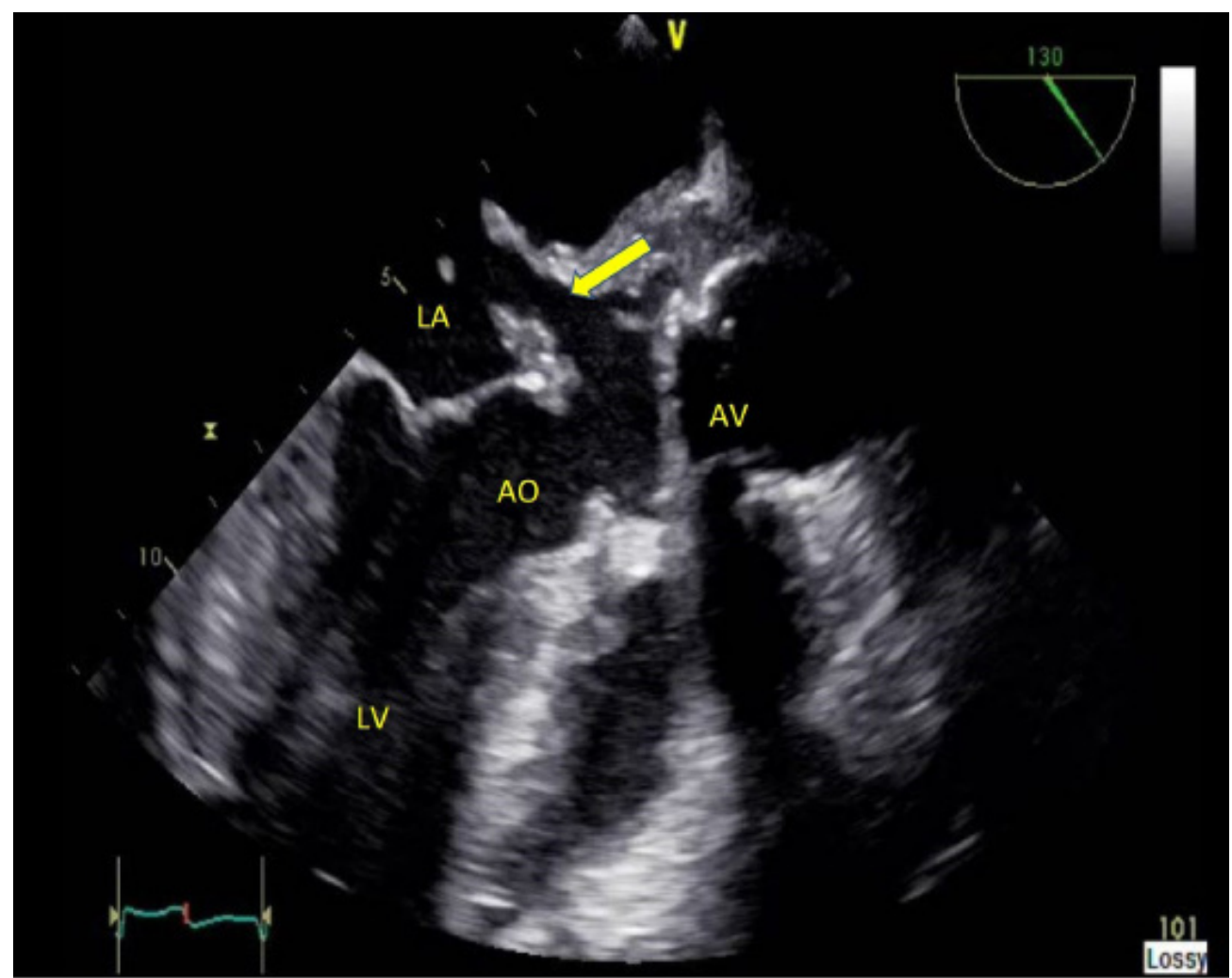

and possible abscess was reported with no evidence of dissection. Infectious disease was consulted and adjusted antimicrobial therapy due to persistent MRSA bacteremia. After evaluation, the cardiothoracic surgeon decided that the patient was a poor surgical candidate and recommended to continue antibiotics and consult palliative care.

The patient's clinical condition began to deteriorate and show signs of acute CHF. He was transferred to the critical care unit due to worsening respiratory failure.

Figure 1: Transesophageal echocardiogram view 1: LA left atrium, LV left ventricle, fistula between aorta and left atrium (yellow arrow). aortic valve endocarditis.

After cardiothoracic surgery evaluation, the TTE was repeated, which showed a fistula between the LVOT, proximal to AOV, and left atrium. The patient had a normal EF but the mechanical aortic valve was noted to have moderate to severe stenosis without regurgitation. A small aortic vegetation was also reported. For further evaluation, a TEE was performed which revealed restricted rocking, suggestive of valve dehiscence with moderate aortic regurgitation, and a large fistula between the aorta and left atrium (Figure 1-2). Also, a valve vegetation with a possible thrombus was noted. An aortic root vegetation

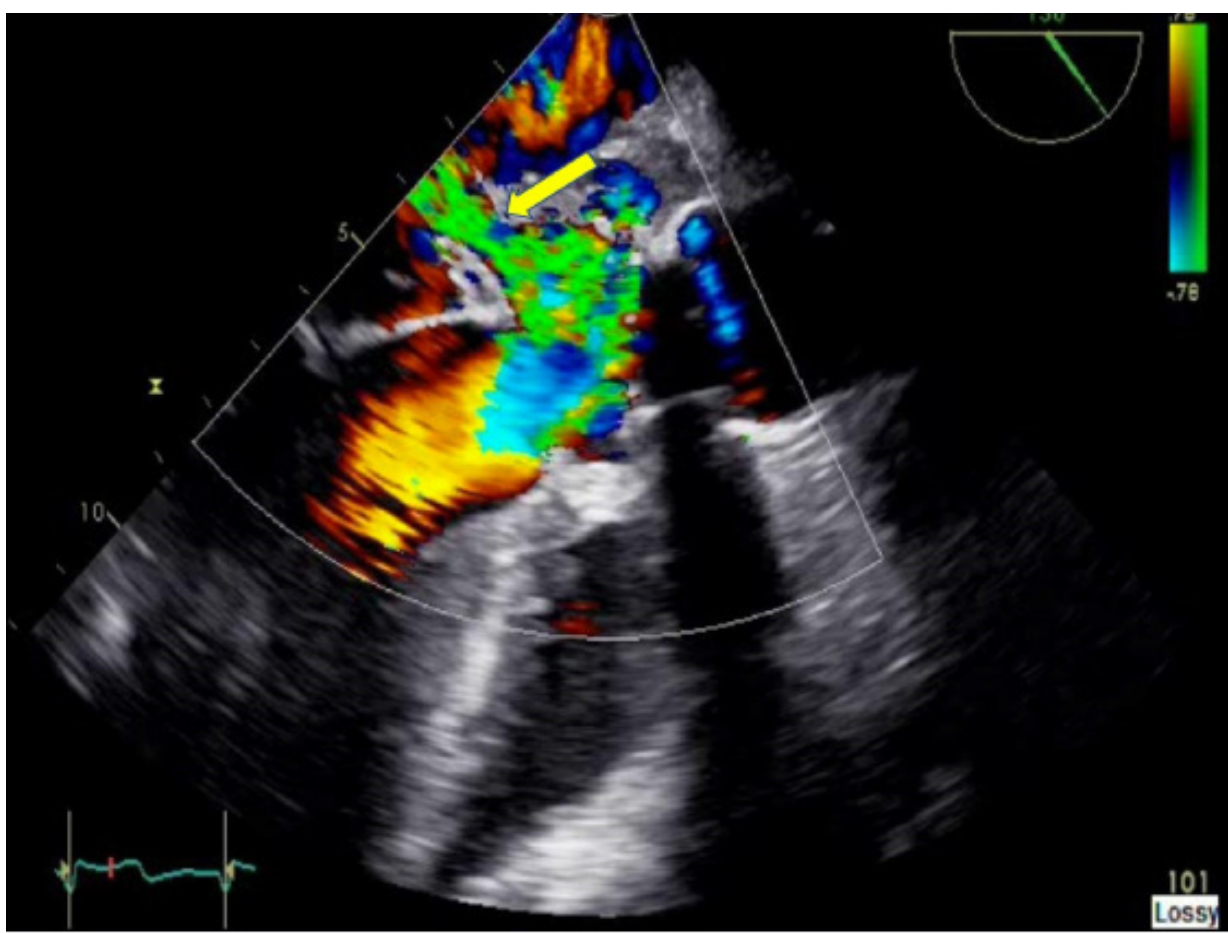

Figure 2: Transesophageal echocardiogram view 2: doppler color flow from the aorta to the left atrium through the fistula (Yellow arrow). mds.marshall.edu/mjm

(C) 2021 Marshall Journal of Medicine
Marshall Journal of Medicine

Volume 7 Issue 2 
due to large pleural effusions. However, the patient continued to decline, was given comfort care, and passed away.

\section{DISCUSSION}

An aorto-atrial fistula, caused by infective endocarditis, is due to the spread of the bacterial infection to the peri-valvular tissue. This leads to the destruction of the tissue and weakness of the wall integrity of the aorta and atrium, causing fistula formation. ${ }^{3}$ In a retrospective multicenter study, aorto-cavity fistula (ACF) is diagnosed in $1.6 \%$ of all cases of IE either identified by echocardiography or during surgery. A fistula was found in $1.8 \%$ of cases of native valve $\mathrm{IE}$, in $3.5 \%$ of prosthetic valves IE from the general population, and in $0.4 \%$ of IV drug abusers. Fistula formation only occurred in the aortic valves with IE and more frequently in prosthetic valves than native valves ( 5.8 vs. $3.6 \%$ respectively). ${ }^{3}$

In a retrospective, multi-center study, the most commonly isolated organism in fistula formation was the staphylococcus species, as seen in $58 \%$ of cases with prosthetic valve and $38 \%$ with a native valve. Staphylococcus aureus was the most single common cause of fistula formation among patients with a native valve. Other pathogens included streptococcus spp. (33\%), Enterococcus spp. (5\%), and culture-negative pathogens $(7 \%)^{3}$

Imaging studies play a major role in the assessment of fistula. In a retrospective, descriptive, multi-center study, the TTE detected fistula formation in $53 \%$ of cases, but the TEE detected $97 \%$ of cases, conveying the superiority of TEE over TTE. ${ }^{3}$ AAF usually occurs on the posterior aspect of the aorta, which is an area better delineated with the TEE than the TTE' ${ }^{1}$. Standard antimicrobial therapy and surgical intervention are the cornerstones of treating IE complicated by AAF. A high index of suspicion and early treatment of the fistula is crucial. Heart failure was observed in $62 \%$ of cases, and the in-hospital mortality was $41 \%^{3}$. Heart failure is caused by the hemodynamic abnormality created by the shunt. Left ventricular function, however, can still remain normal. ${ }^{3}$

Although acquired AAF is uncommon in patients with IE, with the increasing rates of IVDU in the US, the incidence of acquired AAF may increase. From
2002 to 2016, the reported rates of IE due to intravenous drug use have doubled. ${ }^{4}$ This case is unique, as the patient already had a congenital defect of his aortic valve requiring mechanical valve replacement, which was then complicated by IE from his IVDU. Patients with an intravenous substance use disorder, or with a history of underlying congenital heart disease or previous cardiac surgery who develop IE are more likely to have an elevated risk of developing AAF with an increased risk of adverse outcomes.

\section{CONCLUSION}

An aorto-atrial fistula as the result of infective endocarditis is an uncommon complication. Early diagnosis, proper antibiotic treatment, and surgical intervention are crucial steps due to high morbidity and mortality rate. ${ }^{5}$

\section{AUTHOR AFFILIATIONS}

1. Saint Mary's Medical Center, Huntington, West Virginia

2. Lincoln Primary Care Center, Hamlin, West Virginia

3. Saint Joseph Hospital, Lexington, Kentucky

\section{REFERENCES}

1. Ananthasubramaniam K. Clinical and echocardiographic features of aorto-atrial fistulas. Cardiovasc Ultrasound. 2005;3(1):1.

2. Jainandunsing JS, Linnemann R, Maessen J, Natour NE, Lorusso R, Gelsomino S, et al. Aorto-atrial fistula formation and therapy. J Thorac Dis. 2019;11(3):1016.

3. Anguera I, Miro JM, Vilacosta I, Almirante B, Anguita M, Muñoz P, et al. Aorto-cavitary fistulous tract formation in infective endocarditis: clinical and echocardiographic features of 76 cases and risk factors for mortality. Eur Heart J. 2005;26(3):288-97.

4. Kadri AN, Wilner B, Hernandez A V, Nakhoul G, Chahine J, Griffin B, et al. Geographic trends, patient characteristics, and outcomes of infective endocarditis associated with drug abuse in the United States from 2002 to 2016. J Am Heart 
Assoc. 2019;8(19):e012969.

5. Agrawal A, Amor MM, lyer D, Parikh M, Cohen M. Aortico-left atrial fistula: a rare complication of bioprosthetic aortic valve endocarditis secondary to Enterococcus faecalis. Case reports Cardiol. $2015 ; 2015$. 